\title{
Dielectric model of point charge defects in insulating paraelectric perovskites
}

\author{
V. Buniatian, ${ }^{1}$ N. Martirosyan, ${ }^{1}$ A. Vorobiev, ${ }^{2}$ and S. Gevorgian ${ }^{2}$ \\ ${ }^{1}$ Department of Microelectronics, State Engineering University of Armenia, 0009 Yerevan, Armenia \\ ${ }^{2}$ Department of Microtechnology and Nanoscience, Chalmers University of Technology, 41296 Gothenburg, \\ Sweden
}

(Received 17 May 2011; accepted 8 October 2011; published online 15 November 2011)

\begin{abstract}
Some point defects (i.e., oxygen vacancies) create deep trapping levels in the bandgap of the paraelectric phase ferroelectric crystals. Under applied DC field the traps release electrons via the Poole-Frenkel mechanism and become charged. The electric field of a point charge polarizes the crystal locally reducing its permittivity. In this paper a simple theory is proposed for calculating the DC field dependent apparent (measureable) permittivity of a paraelectric crystal with point charge defects. It is shown that the apparent permittivity of a paraelectric crystal may be sufficiently lower as compared with the defectless crystal. This reduction is in addition to the possible reduction of the apparent permittivity associated with the interfacial "dead" layers and strain. (C) 2011 American Institute of Physics. [doi:10.1063/1.3660376]
\end{abstract}

\section{INTRODUCTION: NEGATIVE EFFECTS OF THE OXYGEN VACANCIES IN AGILE MICROWAVE DEVICES}

The point charge defects in an isolating perovskite paraelectric have substantial negative impact on the performance of the devices. Investigations of the electrical and optical properties of $\mathrm{SrTiO}_{3}$ (STO) thin films show, that there is a series of deep-level trapping states with energies in the range of $\mathrm{W}_{\mathrm{v}}+2.4 \mathrm{eV}$ to $\mathrm{W}_{\mathrm{v}}+3.15 \mathrm{eV}$ and a series of shallower traps near the conduction band edge in the range of $\mathrm{W}_{\mathrm{c}}-\mathrm{W}_{\mathrm{t}}$ $=0.06-0.4 \mathrm{eV}$. These electron traps are attributed to oxygen vacancy or iron transition-metal/oxygen vacancy defects. The point charge concentration ranges from $10^{14}$ to $10^{18} \mathrm{~cm}^{3}$ in the as-grown films. Oxygen vacancies are the most common in perovskites. ${ }^{2}$ In agile microwave devices (i.e., varactors, film bulk acoustic wave resonators, FBARs) based on paraelectric phase ferroelectrics, the oxygen vacancies cause high RF and DC leakages currents, microwave losses, ${ }^{3}$ and distortion (hysteresis) of $\mathrm{C}-\mathrm{V},{ }^{4,5} \tan \delta-\mathrm{V},{ }^{6}$ and I-V (Ref. 7) dependencies. In memory applications the oxygen vacancies cause fatigue. ${ }^{8}$ Perhaps the point charge defects should be kept responsible for $1 / \mathrm{f}$ noise too.

The hysteresis effects (non-return capacitance) in paraelectric single crystal STO are explained by excess bulk (volume) charges. ${ }^{5}$ More recently the hysteresis effects are explained by dynamics of the point charges (i.e., oxygen vacancies) at electrode-ferroelectric interfaces. In spite of extensive discussions of this issue, to the best of the author's knowledge, no models of dielectric permittivity including charged point defects in the bulk (bulk traps) of the paraelectrics are reported. In this work a simple model of the point charges (i.e., oxygen vacancy) in an isolating paraelectric phase ferroelectric is considered. It is assumed that without externally applied electric field the vacancy is neutral due to trapped electrons. Under applied external electric field the electrons are de-trapped and the electric field of the charged vacancy polarizes the surrounding crystal locally reducing its permittivity. Analytic approximations for the DC bias dependent apparent dielectric permittivity of the crystal with these locally reduced permittivity regions are proposed.

\section{PHYSICAL MODEL OF A CHARGED POINT DEFECT IN AN ISOLATING PARAELECTRIC PEROVSKITE}

In the experiments with pulsed tuning of ferroelectric varactors $^{9}$ the increased relaxation times $(>100 \mathrm{~s})$, after the threshold fields about $15 \mathrm{~V} / \mu \mathrm{m}$, are clearly associated with the Poole-Frenkel emission of the charge carriers and generation of charged defects with rather long carrier capture times. It is suggested, based on a separate experiment ${ }^{10}$ that the UV illumination generates excess charge carriers that screen these charged defects. Thus, these experiments show that in a good quality insulting paraelectric film the concentration of the background charge carriers is not sufficient (without UV illumination) for screening of the charged defects.

The screening, even though possible, does not seem to be the main mechanism of reduction of the long relaxation time. An alternative interpretation, based on trapping/ de-trapping mechanism, is proposed in this work. The excess carriers increase the trapping rate thereby decreasing the number of charged defects and hence the relaxation time. The extreme relaxation times (hours and days) associated with migration of the charged vacancies (i.e., oxygen vacan$\operatorname{cies}^{11}$ ) are not considered here. The relatively high relaxation times ${ }^{9,10}$ may be explained by the high permittivity, $\varepsilon$, and low conductivity, $\sigma$, of the insulating paraelectric characterized by the Maxwell relaxation time $\tau_{\mathrm{M}}=\varepsilon \varepsilon_{\mathrm{o}} / \sigma=\varepsilon \varepsilon_{\mathrm{o}} / \mu \mathrm{qn} \mathrm{c}_{\mathrm{c}}$. Here $\varepsilon_{\mathrm{o}}=8.86 \times 10^{-12} \mathrm{~F} / \mathrm{m}, \mathrm{q}$ is the electronic charge, $\mathrm{n}_{\mathrm{c}}$ and $\mu$ are, respectively, the free electron density and mobility. For example, for pure STO with $\varepsilon=300, \sigma=10^{-12} \mathrm{~S} / \mathrm{m}$ the relaxation time is $\tau_{\mathrm{M}}=2655 \mathrm{~s}$ !

A simplified model of an oxygen vacancy in a cubic perovskites is shown in Fig. 1. Finding the distribution of the electric field and permittivity around a charged point defect is essentially a three dimensional (3D) problem. It may be solved using modern computer intensive methods like density 


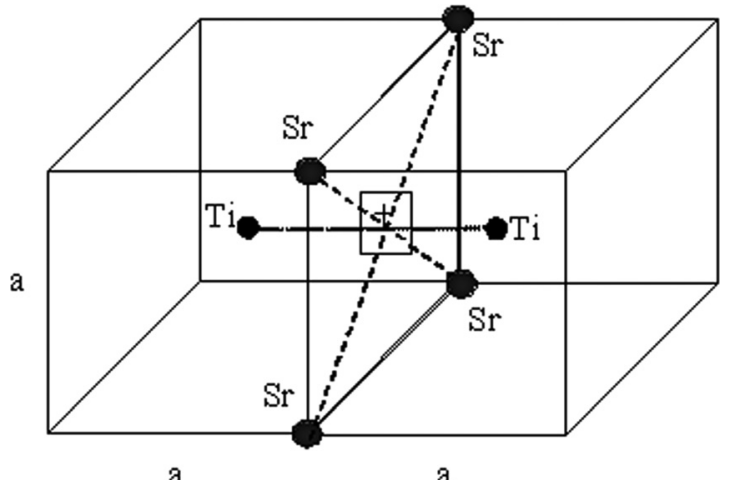

FIG. 1. Charged oxygen vacancy in the (100) plane of the model $\mathrm{SrTiO}_{3}$. Shown are the charged vacancy and neighboring ions only.

function method (DFM). ${ }^{12}$ Here an attempt is made to reduce the $3 \mathrm{D}$ problem into simple $2 \mathrm{D}$ and $1 \mathrm{D}$ problems allowing development of an analytic approximation for the distribution of the dielectric permittivity surrounding the charged point defect. First, a charged defect in the absence of the external electric field is considered to evaluate the internal field in its surrounding. Next, the Poole-Frenkel de-trapping mechanism is assumed to evaluate the 1D local field along the externally applied electric field. Then these field distributions are used to calculate permittivity distributions along the external field and in the plane perpendicular to it. As an example, oxygen vacancies in the STO are considered.

\section{POINT CHARGE DEFECT WITHOUT EXTERNAL FIELD}

Below the activation temperature, $\mathrm{T}<\mathrm{T}_{\mathrm{a}}$, and without external electric field the oxygen vacancy in the STO is neutral $^{13}$ (due to trapped electrons) and causes elastic deformation of the lattice. In the energy diagram is characterized by a deep level $\mathrm{W}_{\mathrm{t}}$, Fig. 2(a). Above the activation temperature the electrons are de-trapped and the vacancy is positively charged. It causes polarization of the surrounding lattice (Fig. 2(b)) and local bending of the conduction band (Fig. 2(c)).

In general, the electric field around has a complex space distribution judging from the locations of the ions in the unit cell of the crystal, Fig. 1. For simplicity a spherical symmetry is assumed where no external field is applied. The point charge may be screened by free electrons or by the polarization field surrounding it. In a paraelectric with some finite conductivity (concentration of free electrons $n_{c}$ ) the screening radius (electron cloud) is given by Debye length,

$$
\mathrm{L}_{\mathrm{D}}(\mathrm{E})=\sqrt{\frac{\varepsilon(\mathrm{E}) \varepsilon_{\mathrm{o}} \mathrm{kT}}{\mathrm{q}^{2} \mathrm{n}_{\mathrm{c}}}}
$$

Due to the very low $n_{c}$ and high permittivity the screening length is very large. This case is not considered in this work. In an isolating paraelectric, a point charge, i.e., oxygen vacancy, is screened by the polarization field. The treatment of this problem requires solution of the Poisson's equation. In contrast to a linear homogeneous dielectric, in a paraelectric the point charge is in the inhomogeneous polarized medium created by the electric field $\left(\mathrm{E}_{\text {int }}\right)$ of the charge itself. The Poisson equation in this case takes the form,

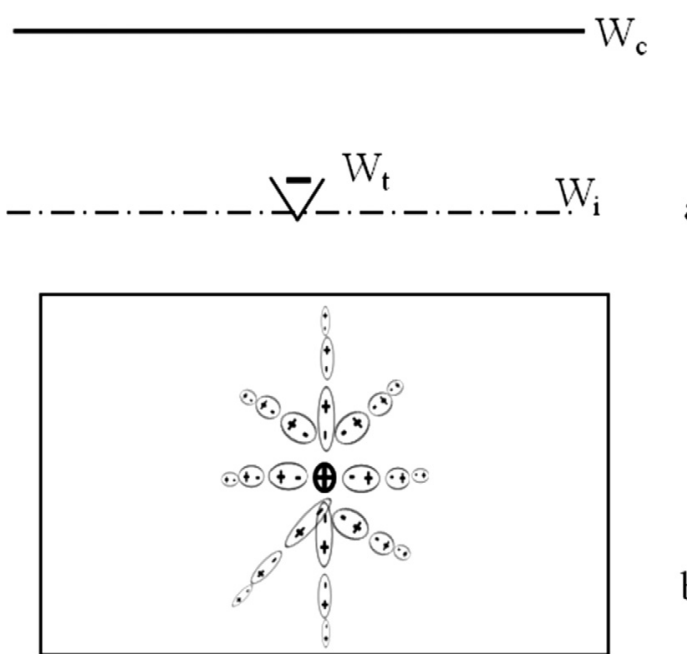

a)

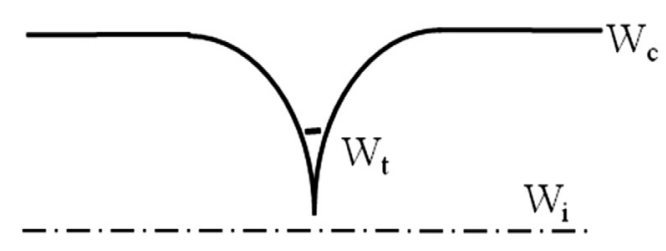

c)

FIG. 2. Oxygen vacancy in a paraelectric perovskite without external electric field.

$$
-\nabla \cdot \nabla \varphi(\mathrm{r})=\nabla \mathrm{E}(\mathrm{r})=\frac{\rho(\mathrm{r})}{\varepsilon_{0} \varepsilon(\mathrm{E}, \mathrm{r})} .
$$

In this equation $\rho(\mathrm{r})$ is the charge density in $\mathrm{C} / \mathrm{m}^{3}$, and the permittivity is field dependent. For a simple case the permittivity is given as

$$
\varepsilon(\mathrm{E}, \mathrm{r})=\frac{\varepsilon(0)}{1+\mathrm{AE}^{2}}
$$

Here $\mathrm{A}=3 \beta\left(\varepsilon_{\mathrm{o}} \varepsilon(0)\right)^{3}$ and $\varepsilon(0)$ is the permittivity at zero bias. For $\mathrm{SrTiO}_{3}, \beta=8 \times 10^{9} \mathrm{~V} \cdot \mathrm{m}^{5} / \mathrm{C}^{3}, \varepsilon(0)=300$, and $\mathrm{A}=0.45$ $\times 10^{-15}(\mathrm{~m} / \mathrm{V})^{2}$. For the considered point charge the charge density may be represented in the form of a delta function or as a Gaussian distribution,

$$
\rho(\mathrm{r})=\frac{Q}{\sigma^{3}\left(\sqrt{(2 \pi))^{3}}\right.} \exp \left[-\frac{\mathrm{r}^{2}}{2 \sigma^{2}}\right]
$$

where $\sigma$ is standard deviation, $\mathrm{Q}$ is the charge. Using Eqs. (2)-(4) leads to the following form of the Poisson's equation:

$$
\nabla \mathrm{E}=\frac{1+\mathrm{AE}^{2}}{\varepsilon_{0} \varepsilon(0)} \frac{Q}{\sigma^{3}[\sqrt{(2 \pi)}]^{3}} \exp \left[-\frac{\mathrm{r}^{2}}{2 \sigma^{2}}\right] .
$$

Using the method of separation of variables one arrives at the following $1 \mathrm{D}$ solution:

$$
\mathrm{E}_{\text {int }}(\mathrm{x})=\frac{1}{\sqrt{\mathrm{A}}} \tan \left[\frac{\sqrt{\mathrm{A}}}{\varepsilon_{0} \varepsilon(0)} \frac{Q}{4 \pi \sigma^{2}} \operatorname{erfc}\left(\frac{\mathrm{x}}{\sqrt{2} \sigma}\right)\right] .
$$

Index int indicates the internal electric field created by the point charge itself, as opposed to the externally applied field 

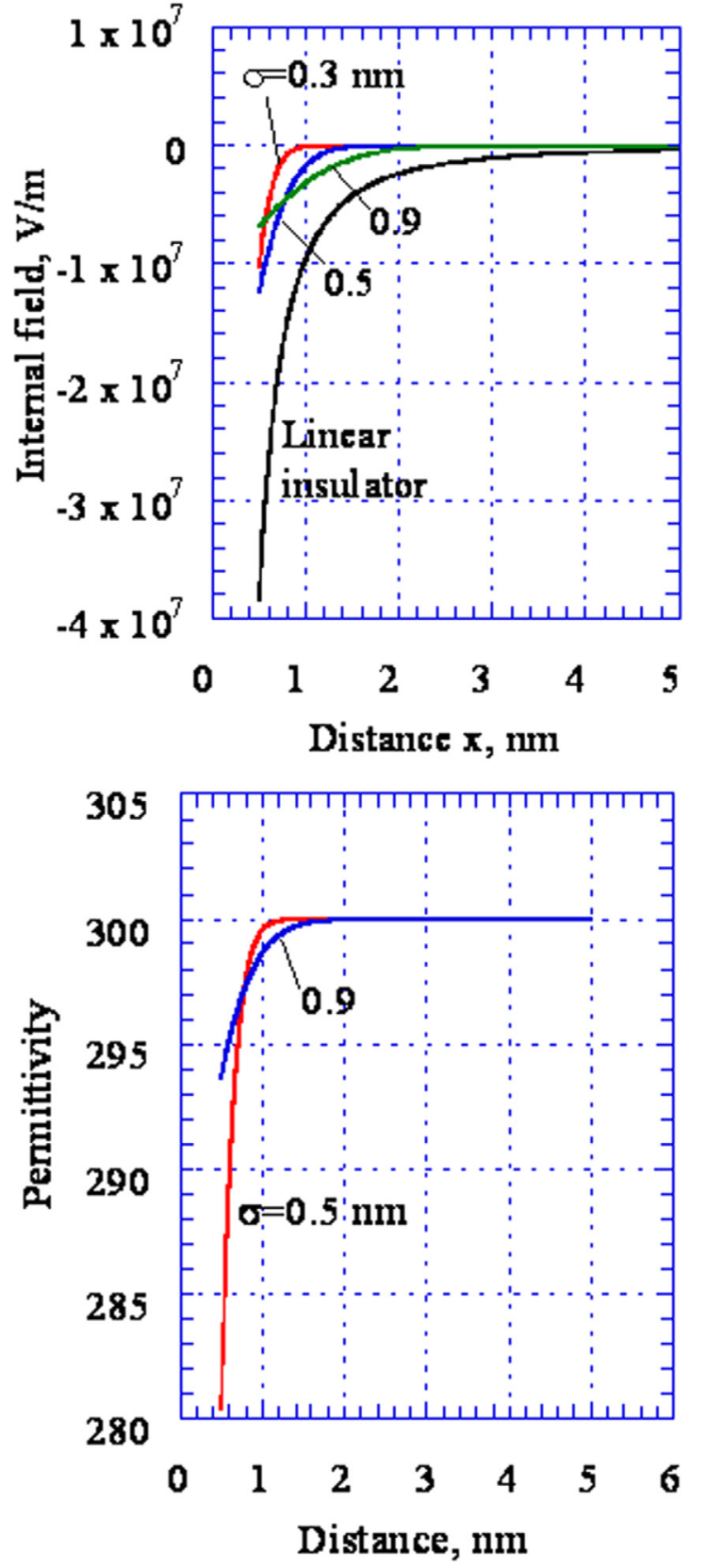

FIG. 3. (Color online) Field (a) and permittivity (b) distributions around a point charge in STO.

considered below. Figure 3(a) depicts the internal field distribution around point charge $\mathrm{Q}=2 \mathrm{q}$ in the STO. The field distribution of a charge $\mathrm{Q}$ in an ordinary linear dielectric with no field dependent permittivity, $\mathrm{E}(\mathrm{x})=\mathrm{Q} /\left(4 \pi \varepsilon \varepsilon_{0} \mathrm{x}^{2}\right)$, is also given for $\varepsilon=300$. In both cases the fields decay rapidly away from the point charge and becomes close to zero at some distance from it. This is the distance where the field of the point charge is practically compensated (screened) by the field of the induced dipoles. The screening length is shorter and the field reduction is sharper for the nonlinear dielectric. In contrast to an ordinary linear insulator, in a nonlinear insulator the field of the point charge reduces the permittivity which leads to reduction of the screening radius. In other words most of the "voltage" (field induced by point charge) drops on the low permittivity regions closer to the point charge.

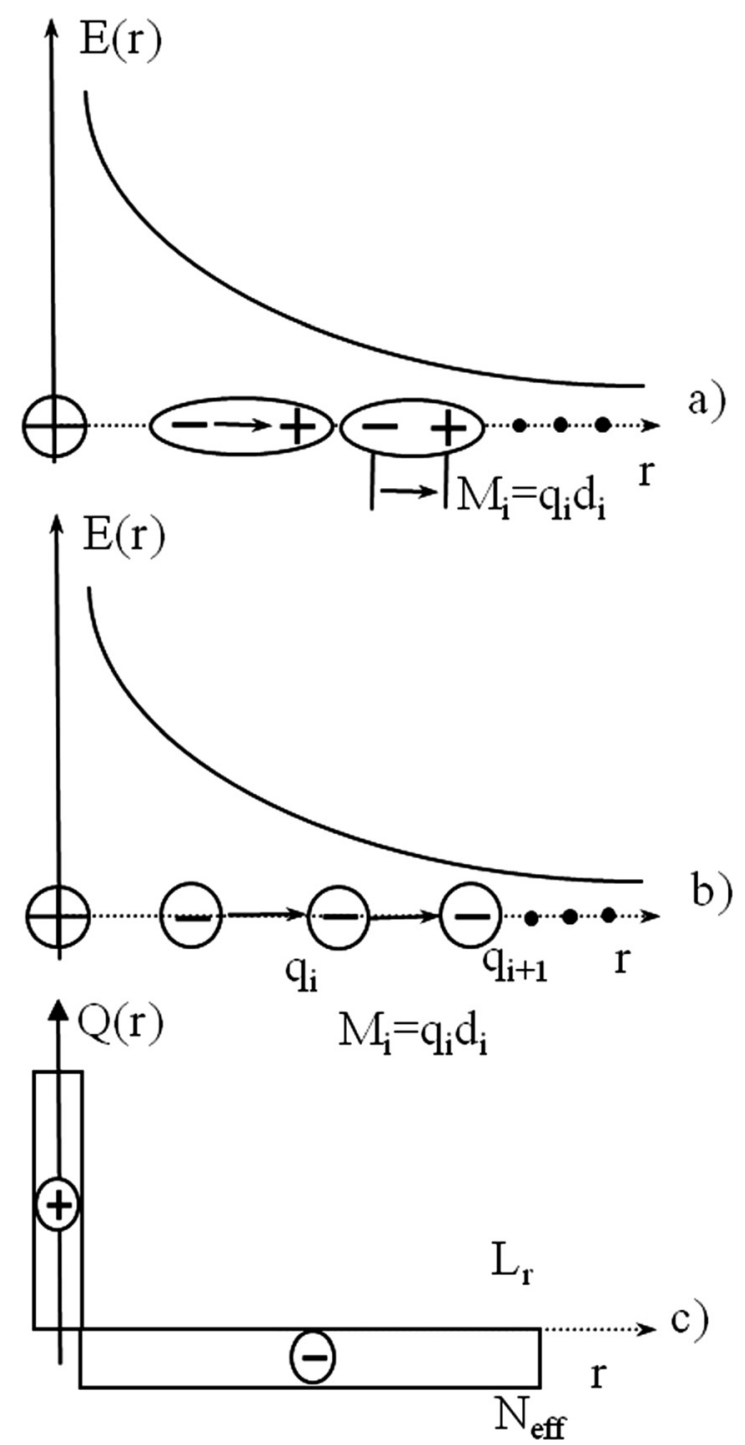

FIG. 4. Induced dipoles (a), dipoles due to fictitious charges (b), and space charge approximation (c).

Figure 3(b) shows the local permittivity distribution around a charged defect in the STO calculated using Eqs. (6) and (3).

The screening radius may be estimated by assuming fixed (not movable) fictitious "polarization charges" associated with the induced dipoles. The dipole moments $\mathrm{M}_{\mathrm{i}}=\mathrm{q}_{\mathrm{i}} \mathrm{d}_{\mathrm{i}}$, Fig. 4(a), decrease away from the point charge $\left(M_{i}=0\right.$ at $\left.r=\infty\right)$. Formally the same electric field and dipole moment distribution may be generated by fictitious negative charges, $\mathrm{q}_{\mathrm{i}}$, such that $\left|q_{i+1}\right|<\left|q_{i}\right|$ (Fig. 4(b)). And then the point charge and its polarized surrounding may be represented in the space charge approximation (Fig. 4(c)) where the point charge Q is screened by a sphere of negative charges with a radius,

$$
\mathrm{L}_{\mathrm{r}}\left(\mathrm{E}_{\text {int }}\right)=\sqrt{\frac{\varepsilon\left(\mathrm{E}_{\text {int }}\right) \varepsilon_{\mathrm{o}} \mathrm{kT}}{\mathrm{Q}^{2} \mathrm{~N}_{\mathrm{eff}}}},
$$

where $N_{\text {eff }}$ is the concentration of the fictitious polarization charge in a sphere with radius $L_{r}$ surrounding the point charge, Fig. 2(b). The absolute amount of this negative charge is the same as the total charge of the oxygen vacancy after de-trapping. 
The effective concentration of the polarization charges in the sphere of radius $L_{r}$ is

$$
\mathrm{N}_{\text {eff }}=\frac{\mathrm{i}}{\frac{3}{4} \pi \mathrm{L}_{\mathrm{r}}^{3}} .
$$

For neutral, single, and double charged oxygen vacancies, respectively, $i=0,1,2$. Only $i=2$ is considered in this paper. Using Eq. (8) in Eq. (7) results in

$$
\mathrm{L}_{\mathrm{r}}\left(\mathrm{E}_{\mathrm{int}}\right)=\frac{4}{3} \frac{\mathrm{Q}^{2}}{\pi \varepsilon\left(\mathrm{E}_{\mathrm{int}}\right) \varepsilon_{\mathrm{o}} \mathrm{kT}} .
$$

For an oxygen vacancy in the STO with $\mathrm{Q}=2 \mathrm{q}$ using Eqs. (3), (6), and (9) at $\mathrm{E}_{\mathrm{int}}=0$ limit yields $\mathrm{L}_{\mathrm{r}} \approx 2.0 \mathrm{~nm}$, which is in a good agreement with the field distribution shown in Fig. 3(a).

\section{CHARGING THE POINT DEFECT BY POOLE-FRENKEL DE-TRAPPING}

Figures 5(a) and 5(b) illustrate the structure of a paraelectric crystal including a neutral oxygen vacancy and the conduction band at relatively low external fields.

At applied fields, where qE exceeds the activation energy the electrons are de-trapped. The vacancy gets charged, polarizing the crystal predominantly along the applied field $(x$, Fig. $5(\mathrm{c})$ ). The deformation of the conduction band, distribution of the charges and magnitude of the electric field are shown in Figs. 5(d)-5(f). Following Lapascu ${ }^{14}$ it is assumed that the component of the applied electric field normal to the $\mathrm{x}$ axis (caused by the induced anisotropy of the permittivity) is negligible. In other words in the plane perpendicular to the direction of the applied field the local field distribution is still given by Eq. (6) and along the $\mathrm{x}$ axis (direction of the external electric field) it is characterized by a cylindrical symmetry.

Since the argument of the tan function in Eq. (6) is small the field distribution may be simplified to

$$
\mathrm{E}_{\mathrm{int}} \cong \frac{\mathrm{Q}}{4 \pi \varepsilon_{0} \varepsilon(0) \mathrm{x}_{\mathrm{e}}^{2}}
$$

where the effective distance from the point charge is given by

$$
\mathrm{x}_{\mathrm{e}}^{2}=\frac{\sigma^{2}}{\operatorname{erfc}\left(\frac{\mathrm{x}}{\sqrt{2} \sigma}\right)} .
$$

The form of Eq. (10) is similar to the field distribution induced by a point charge $\mathrm{Q}$ in an ordinary linear dielectric, $\mathrm{E}(\mathrm{x})=\mathrm{Q} /\left(4 \pi \varepsilon \varepsilon_{\mathrm{o}} \mathrm{x}^{2}\right)$. However, the effective distance $\mathrm{x}_{\mathrm{e}}$ changes much faster than $\mathrm{x}$ itself. Under an applied $\mathrm{x}$-direction external electric field $E_{\text {ext }}$ the local field distribution in the vicinity of the charged defect is

$$
\mathrm{E}_{\mathrm{loc}}(\mathrm{x})=\mathrm{E}_{\mathrm{int}}(\mathrm{x}) \pm \mathrm{E}_{\mathrm{ext}},
$$

where "+" is taken for the field on the left and "-"on the right sides of the defect corresponding to the graphical presentation in Fig. 5. In general the internal $\mathrm{E}_{\text {int }}$ field is given

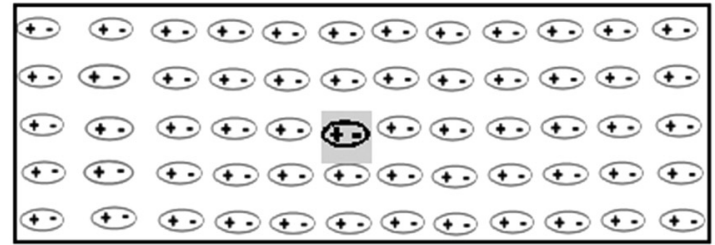

a)

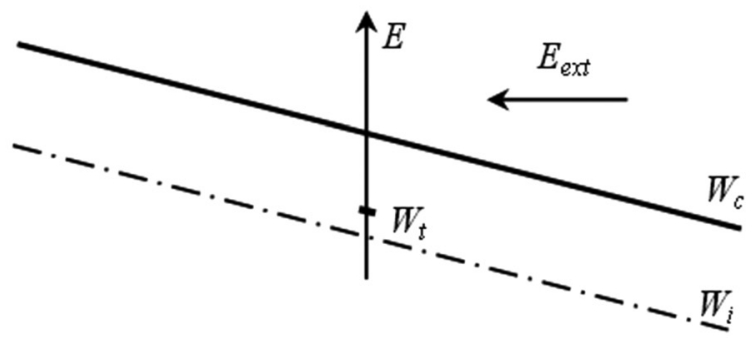

b)

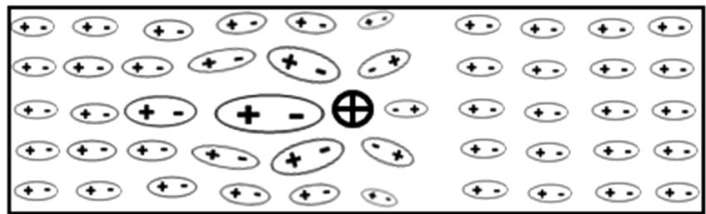

c)

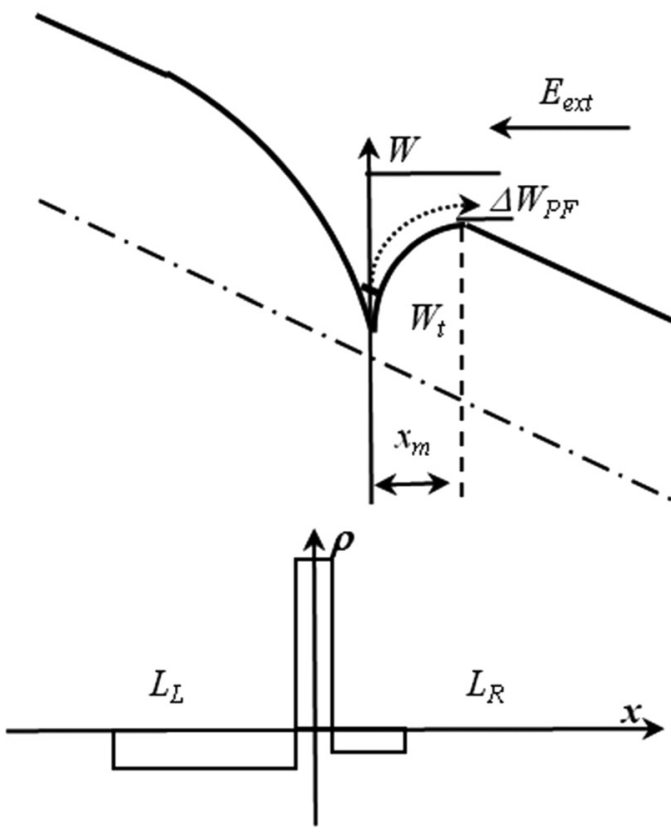

d)

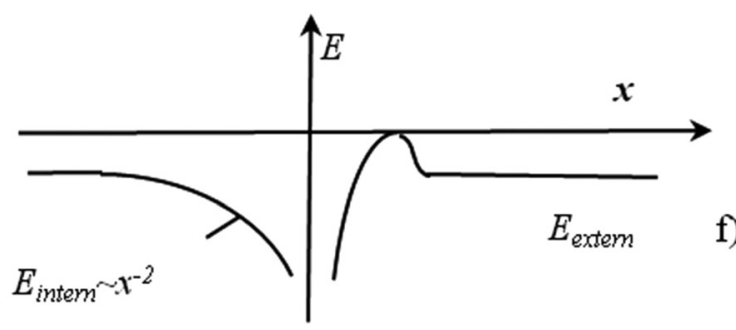

FIG. 5. Oxygen vacancy in an isolating paraelectric perovskite below activation energy $(a, b)$, and under externally applied electric field with $\mathrm{qE}$ exceeding Poole-Frenkel activation energy (c, d, e, f).

by Eq. (6) but Eq. (10) is a rather good approximation. The distribution of the permittivity under external field is

$$
\varepsilon(\mathrm{E}, \mathrm{x})=\frac{\varepsilon(0)}{1+\mathrm{A}\left[\mathrm{E}_{\mathrm{loc}}(\mathrm{x})\right]^{2}} .
$$

The local field distribution on the left-hand side of the charged vacancy calculated by Eq. (10) is shown in 

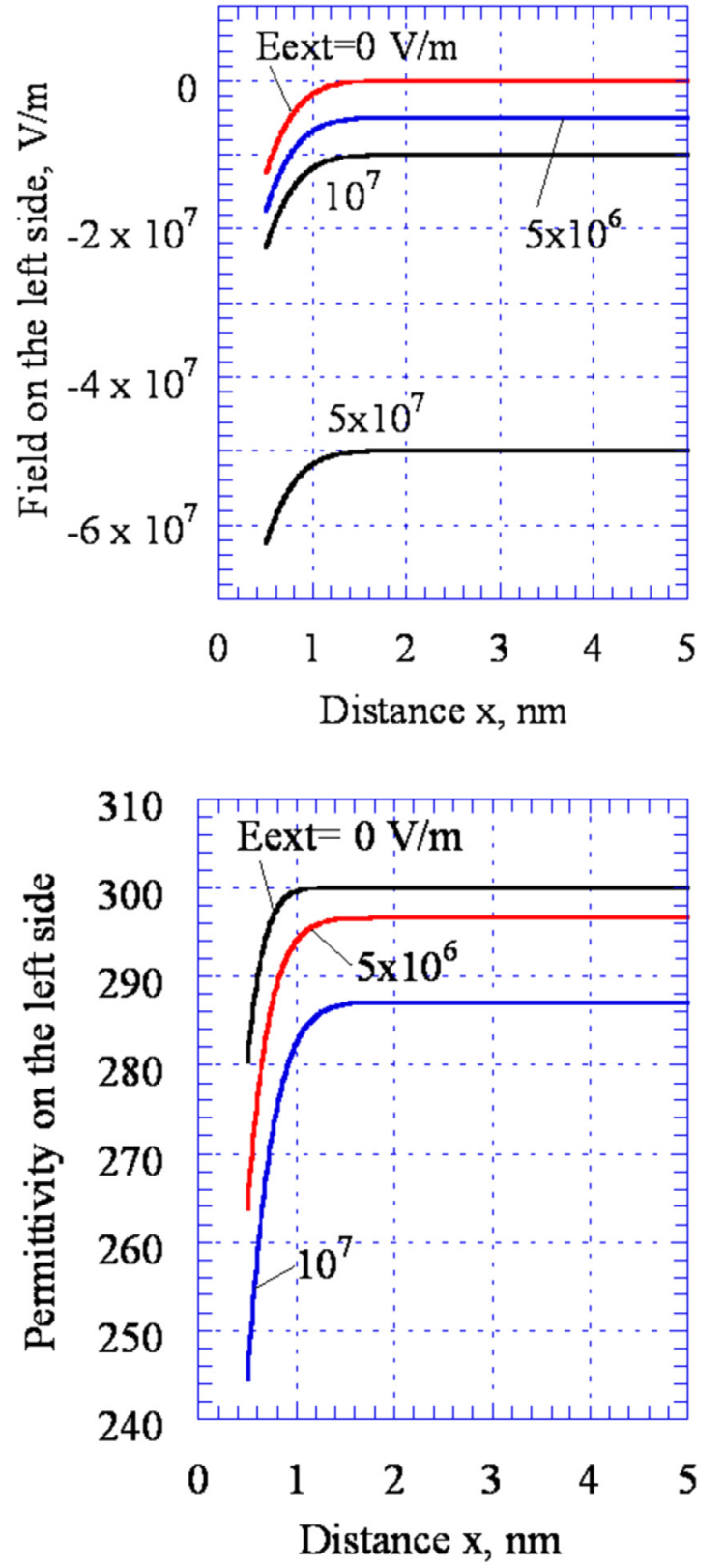

FIG. 6. (Color online) Local field (a) and permittivity (b) distributions on the left side of the point charge.

Fig. 6(a). The distribution (10) is used in Eq. (13) for calculation of the permittivity distribution depicted in Fig. 6(b). As expected, it decreases going toward the charged defect. At the same time it is constant at some distance $\mathrm{L}_{\mathrm{L}}$ away from the charged vacancy where it decreases with increasing external bias as in the STO without charged defects. Below the distance $\mathrm{L}_{\mathrm{L}}$ the internal field dominates resulting rapid decrease in permittivity. As a first approximation $L_{L}$ may be estimated using the space charge formality considered above. The external field expands the length of the space charge region. Using Eq. (9) its expansion may be given by

$$
\mathrm{L}_{\mathrm{L}}=\frac{4}{3} \frac{\mathrm{Q}^{2}}{\pi \varepsilon(0) \varepsilon_{\mathrm{o}} \mathrm{kT}}\left[1+\mathrm{AE}_{\mathrm{loc}}^{2}\right] .
$$

The local field distribution on the right-hand side is shown in Fig. 7(a). At some distance, $L_{R}$, the internal field is fully com-
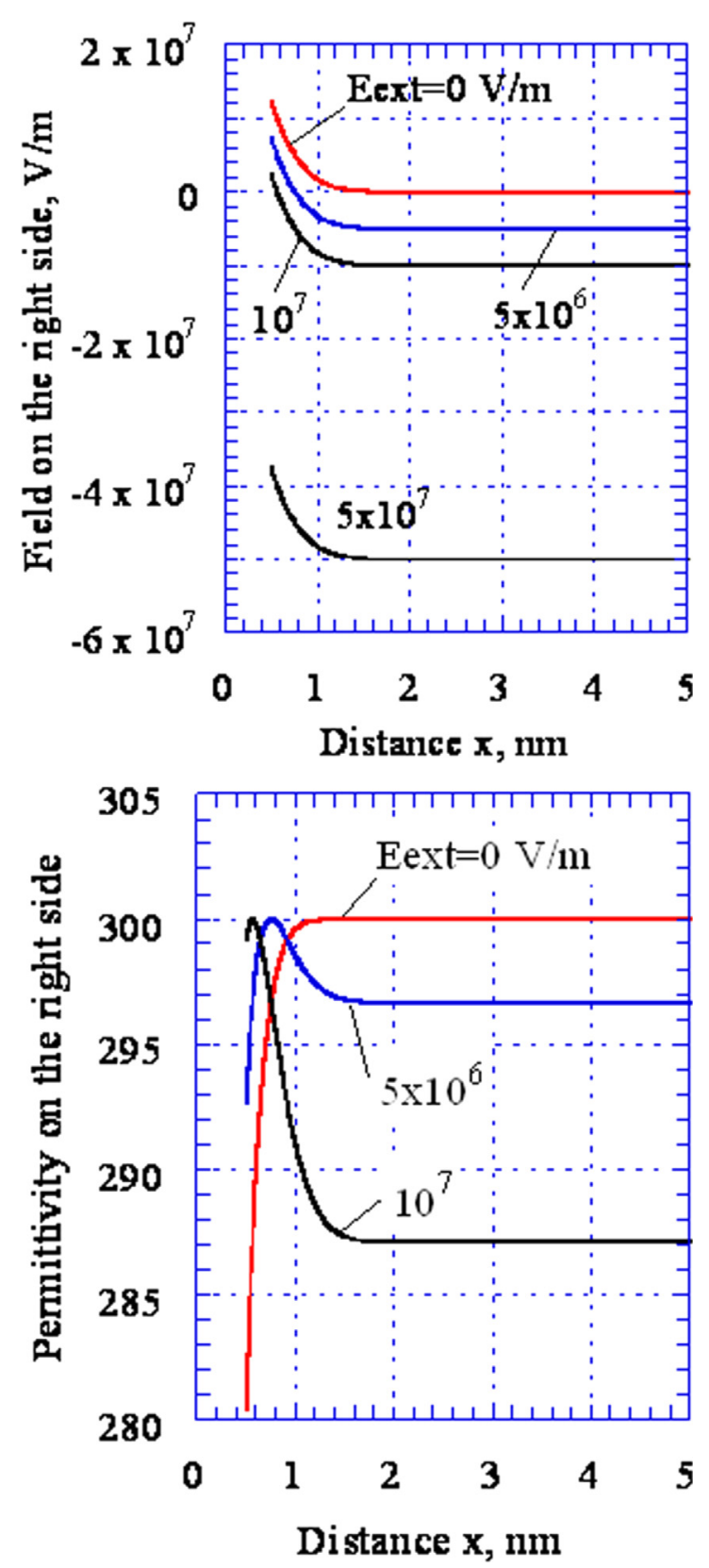

FIG. 7. (Color online) Local field (a) and permittivity (b) distributions on the right side of the point charge.

pensated by an external applied field leading to increased permittivity near the point charge as it is seen in Fig. 7(b). From the condition $\mathrm{E}_{\mathrm{loc}}=0$, Eqs. (10) and (11) one has

$$
\mathrm{L}_{\mathrm{R}}=\sigma \sqrt{2} \operatorname{erf}^{-1}\left[1-\frac{4 \pi \varepsilon_{\mathrm{o}}}{Q} \varepsilon(0) \mathrm{E}_{\mathrm{ext}}\right] .
$$

At this distance the permittivity takes the value $\varepsilon(0)$ as it follows from Eqs. (12), (13), and Fig. 7(b). For $\mathrm{x}<\mathrm{L}_{\mathrm{R}}$ the permittivity decreases rapidly under dominant internal field, while at $\mathrm{x}>\mathrm{L}_{\mathrm{R}}$ the external field dominates. Again, far away from the defect the permittivity is independent of the coordinate $x$ and decreases with increased external bias as in the STO without changed vacancies.

The DC bias dependent screening lengths $\mathrm{L}_{\mathrm{L}}$ and $\mathrm{L}_{\mathrm{R}}$ are shown in Fig. 8. As it is seen from Eqs. (14), (15), and Fig. 8 
increasing $\mathrm{E}_{\text {ext }}$ leads to increased $\mathrm{L}_{\mathrm{L}}$ and reduced $\mathrm{L}_{\mathrm{R}}$. The external field dependence of the total length of the reduced permittivity region around the point charge, $\mathrm{L}_{\mathrm{x}}=\mathrm{L}_{\mathrm{L}}+\mathrm{L}_{\mathrm{R}}$, is shown in Fig. 8(c). Its initial reduction is associated with the reduction of $L_{R}$, Fig. 8(b). After reaching $L_{R}=0$, the
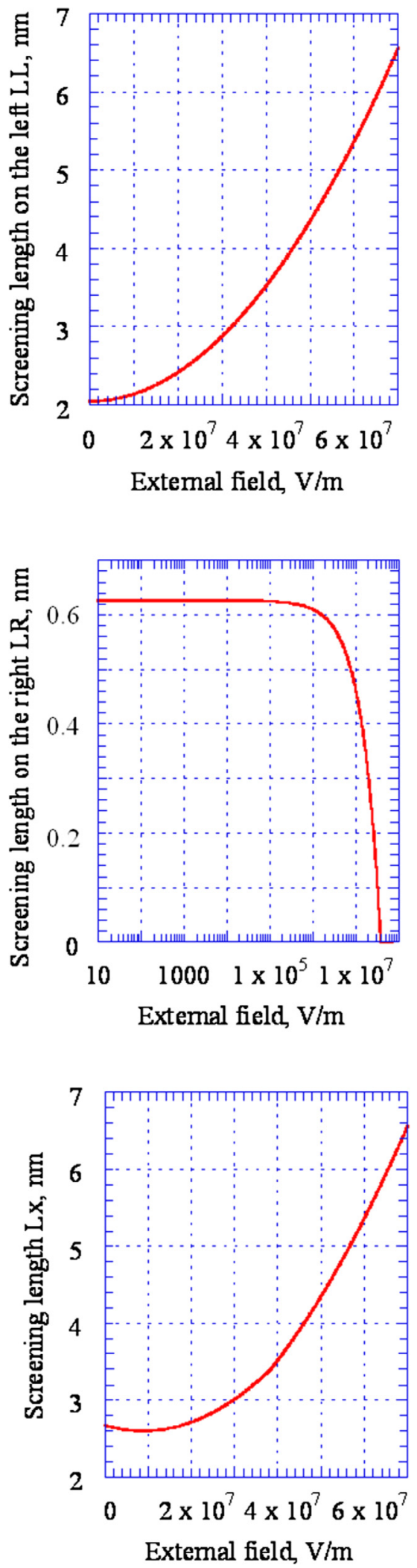

FIG. 8. (Color online) Screening lengths on the left (a), right (b) sides and the total screening length $\mathrm{L}_{\mathrm{x}}(\mathrm{c})$. increase in $\mathrm{L}_{\mathrm{x}}$ is associated with expansion of the $\mathrm{L}_{\mathrm{L}}$. The external filed changes not only the lengths of the polarized regions, but also the permittivity in these regions-considered in the next section.

\section{APPARENT (MEASUREABLE) PERMITTIVITY}

For modeling the effects of trapping/de-trapping on the apparent permittivity an average permittivity of the polarized region is introduced. It follows from Eq. (10) that the electric field at the limit $x=0$ is extremely large and the permittivity tends to be zero. However, calculation of the permittivity in this limit may not be done using Eq. (3) since it is valid for low electric fields. Moreover, the permittivity is not defined for the distance less than the inter-ionic distance in the unit cell. An analytic expression for the average permittivity, $\varepsilon_{C D}$, of the polarized region may be found using a simple linear interpolation: $\varepsilon_{C D}=\left(\varepsilon_{\max }-\varepsilon_{\min }\right) / 2 \approx \varepsilon(\mathrm{E}, \mathrm{x}) / 2$, where $\varepsilon(\mathrm{E}, \mathrm{x})$ is the bias dependent permittivity at $\mathrm{x} \approx \mathrm{L}_{\mathrm{L}}$ and $\mathrm{L}_{\mathrm{R}}$ boundaries. Averaging the permittivities on left and right and considering them as two serially connected capacitors leads to the following average field dependent permittivity:

$$
\varepsilon_{\mathrm{cd}}\left(\mathrm{E}_{\mathrm{ext}}\right) \approx \frac{2 \mathrm{~L}_{\mathrm{L}}\left(\mathrm{E}_{\mathrm{ext}}\right)}{\frac{2 \mathrm{~L}_{\mathrm{R}}}{\varepsilon_{\mathrm{cd}}\left(\mathrm{E}_{\mathrm{ext}}, \mathrm{L}_{\mathrm{L}}\right)}+\frac{\varepsilon_{\mathrm{cd}}\left(\mathrm{E}_{\mathrm{ext}}, \mathrm{L}_{\mathrm{R}}\right)}{} .}
$$

As discussed above, the component of the electric field associated with the expansion of the polarized region in the plane perpendicular to the $\mathrm{x}$-axis may be ignored and the polarized region is represented as a cylinder with radius $L_{r}$ and height $\mathrm{L}_{\mathrm{x}}$, Fig. 9(a), having an average permittivity $\varepsilon_{\mathrm{cd}}$ given by Eq. (16). If $n_{t}$ is the concentration of the point charges, then each
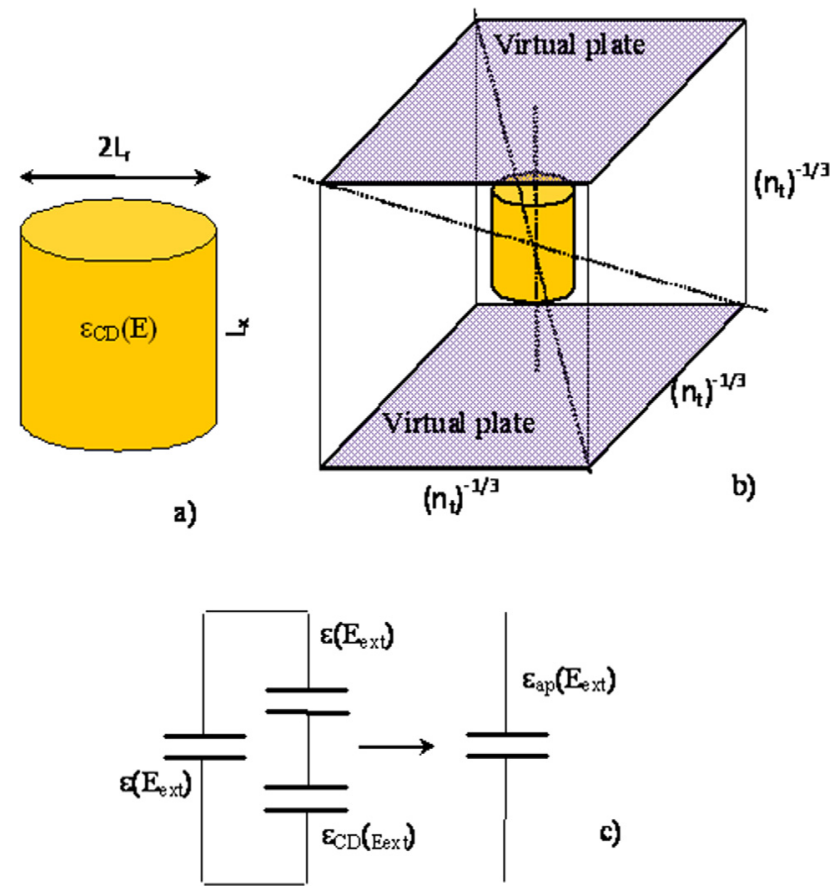

FIG. 9. (Color online) Cylindrical approximation of the polarized region (a), its model in the matrix of a paraelectric (b), and equivalent circuit representation (c). 
of them may be represented by a volume shown in Fig. 9(b). By considering the equivalent capacitances of its different parts, Fig. 9(c), and the total capacitance between the virtual plates the apparent permittivity may be approximated as

$$
\begin{aligned}
& \varepsilon_{\text {ap }}\left(\mathrm{E}_{\text {ext }}\right)=\varepsilon\left(\mathrm{E}_{\text {ext }}\right) \\
& \quad \times\left\{1-\pi \mathrm{L}_{\mathrm{r}}^{2} \mathrm{n}_{\mathrm{t}}^{2 / 3}\left[1+\frac{2 \varepsilon_{\mathrm{cd}}\left(\mathrm{E}_{\mathrm{ext}}\right)}{2\left(1-\mathrm{L}_{\mathrm{x}} \mathrm{n}_{\mathrm{t}}^{1 / 3}\right) \varepsilon_{\mathrm{cd}}+\varepsilon\left(\mathrm{E}_{\mathrm{ext}}\right)}\right]\right\},
\end{aligned}
$$

where $L_{r}$ is given by Eq. (9), $\varepsilon\left(E_{\text {ext }}\right)$ is given by Eq. (3), and the bias depended average permittivity in the vicinity of the charged defect is given by Eq. (16), $\mathrm{L}_{\mathrm{x}}=\mathrm{L}_{\mathrm{L}}+\mathrm{L}_{\mathrm{x}}$. Equation (17) is valid for small filling factor $L_{x} \times\left(n_{t}\right)^{1 / 3}<1$, i.e., where the charged defects do not interact. The concentration of the charged defects is a function of the applied field, $n_{t}=n_{t}\left(E_{\text {ext }}\right)$, and activation energy of the traps. These dependencies are considered in the next section.

\section{DC BIAS DEPENDENCE OF THE CHARGED DEFECT CONCENTRATION}

The dielectric model considered above is within the low frequency (microwave) limit where the permittivity of the paraelectric is associated with the ionic polarization. It is frequency independent below the soft mode frequency. This includes also the polarized surrounding of the point charges, i.e., after de-trapping of the electron. On the other hand the Poole-Frenkel model, considered below, is based on the hydrogen model where the defect with the bounded (trapped) electron is immersed into a dielectric. In this model the point charge with the bounded electron (i.e., before de-trapping) represents an "oscillator" that contributes to the dielectric permittivity in the optical limit, i.e., well above the soft mode. In other words, it is associated with the electronic polarization. Thus, in contrast to the microwave permittivity used in the dielectric model above, in the Poole-Frenkel model considered below, the permittivity is taken in the optical limit $\left(\varepsilon_{\text {opt }}\right)$ and it characterizes only the charge de-trapping process, not the dielectric permittivity at microwave frequencies.

Under an applied external field, $\mathrm{E}_{\text {ext }}$, some electrons are released from the traps. For a single trap with energy $\mathrm{W}_{\mathrm{t}}$ the electron capture, $r_{c E}$, and emission, $r_{e E}$, rates (by the traps) are given by ${ }^{15}$

$$
\begin{gathered}
\mathrm{r}_{\mathrm{cE}}=\mathrm{n}_{\mathrm{c}} \mathrm{N}_{\mathrm{t}}\left(1-\mathrm{f}_{\mathrm{t}}\left(\mathrm{E}_{\mathrm{ext}}\right)\right) \sigma_{\mathrm{c}} \mathrm{v}_{\mathrm{th}}, \\
\mathrm{r}_{\mathrm{eE}}=\mathrm{N}_{\mathrm{t}} \mathrm{f}_{\mathrm{t}}\left(\mathrm{E}_{\mathrm{ext}}\right) \nu \exp \left(-\frac{\mathrm{qW}_{\mathrm{t}}}{\mathrm{KT}}+\beta_{\mathrm{PF}} \sqrt{\mathrm{E}_{\mathrm{ext}}}\right),
\end{gathered}
$$

where

$$
\beta_{\mathrm{PF}}=\frac{1}{\mathrm{kT}} \sqrt{\frac{\mathrm{q}^{3}}{\pi \varepsilon_{\mathrm{o}} \varepsilon_{\mathrm{opt}}}}
$$

$\mathrm{N}_{\mathrm{t}}$ is the total (neutral and charged) density of the point defects, $\sigma_{\mathrm{c}}$ is the capture cross section, $\mathrm{v}_{\mathrm{th}}=\left(3 \mathrm{KT} / \mathrm{m}^{*}\right)^{1 / 2}$ is the thermal velocity of the electrons, $f\left(E_{\text {ext }}\right)$ is the field dependent electron distribution function (probability of an electron having energy $\mathrm{W}_{t}$ ), $\mathrm{n}_{\mathrm{c}}$ is the free electron concentration at the presence of the field. The free electrons are injected from the Schottky barriers (electrodes used for DC bias), and partly from the deep level traps. $\nu$ is the "attempt to escape" frequency which is connected to relaxation time with the relationship,

$$
\tau=\frac{1}{\nu} \exp \left(\frac{\mathrm{qW}_{\mathrm{t}}}{\mathrm{kT}}-\beta_{\mathrm{PF}} \sqrt{\mathrm{E}_{\mathrm{ext}}}\right) .
$$

Practically the optical dielectric permittivity, $\varepsilon_{\text {opt }}$, is independent of the applied DC bias. Note that in Eqs. (19)-(21) the electric field, $\mathrm{E}=\mathrm{E}_{\text {ext }}$, since the permittivity, $\varepsilon_{\text {opt }}$, is associated with the electronic (not ionic) polarization. In steady state conditions $r_{\mathrm{cE}}=\mathrm{r}_{\mathrm{eE}}$ and the field dependent distribution function $\mathrm{f}\left(\mathrm{E}_{\text {ext }}\right)$ becomes

$$
\mathrm{f}_{\mathrm{t}}\left(\mathrm{E}_{\mathrm{ext}}\right)=\frac{\mathrm{n}_{\mathrm{c}} \sigma \mathrm{v}_{\mathrm{th}}}{\mathrm{n}_{\mathrm{c}} \sigma_{\mathrm{c}} \mathrm{v}_{\mathrm{th}}+\nu \exp \left(-\frac{\mathrm{qW}_{\mathrm{t}}}{\mathrm{kT}}+\beta_{\mathrm{PF}} \sqrt{\mathrm{E}_{\mathrm{ext}}}\right)} .
$$

Hence the external field dependent density of the charged vacancies is

$$
\mathrm{n}_{\mathrm{t}}\left(\mathrm{E}_{\mathrm{ext}}\right)=\left[1-\mathrm{f}_{\mathrm{t}}\left(\mathrm{E}_{\mathrm{ext}}\right)\right] \mathrm{N}_{\mathrm{t}}
$$

As it follows from Eqs. (22) and (23), with increasing the external field $\mathrm{f}\left(\mathrm{E}_{\text {ext }}\right)$ decreases leading to an increased density of charged defects which results in a reduction of the apparent permittivity according to Eq. (17).

\section{RESULTS AND DISCUSSIONS}

\section{A. Numerical results}

The materials parameters used in calculations are given in Table I. First, for an estimation of the maximum value of the non-return capacitance (permittivity) the bias dependence of the density of the charged carriers is considered, i.e., in Eq. (17) $n_{t}=$ const is assumed. The non-return permittivity is defined as $\Delta \varepsilon\left(\mathrm{n}_{\mathrm{t}}\right)=\left[\varepsilon(0)-\varepsilon_{\mathrm{ap}}\left(0, \mathrm{n}_{\mathrm{t}}\right)\right] / \varepsilon(0)$. As it is seen from Fig. 10(a) for densities $n_{t}<10^{22} \mathrm{~m}^{-3}$ the effect of the point charges on the apparent permittivity is negligible at all bias fields. The dependence of $\Delta \varepsilon$ on the density of the charged defects is shown in Fig. 10(b). A substantial reduction of the apparent permittivity may be expected due to the charged defects. This is in addition to the possible reductions due to the interfacial"dead" layers ${ }^{16,17}$ and/or misfit strain. ${ }^{18}$

Figure 11 depicts the dependence of the concentration of the charged traps on external applied voltage calculated

TABLE I. Material parameters used in calculations.

\begin{tabular}{lcc}
\hline \hline Parameter & Unit & Value \\
\hline Permittivity at $\mathrm{V}=0, \varepsilon(0)$ & $\mathrm{NA}$ & 300 \\
Nonlinearity coefficient, $\beta$ & $\mathrm{J} \cdot \mathrm{m}^{5} / \mathrm{C}^{4}$ & $8 \cdot 10^{9}$ \\
Optical permittivity, $\varepsilon_{\mathrm{opt}}$ & $\mathrm{NA}$ & 3.8 \\
Capture cross section, $\sigma$ & $\mathrm{cm}^{2}$ & $10^{-16}$ \\
Escape frequency, $\nu$ & $\mathrm{Hz}$ & 0.1 \\
Poole-Frenkel coefficient, $\beta_{\mathrm{PF}}$ & $\mathrm{C} \cdot \mathrm{V}^{1 / 2} / \mathrm{J}$ & $1.5 \cdot 10^{-4}$ \\
\hline \hline
\end{tabular}



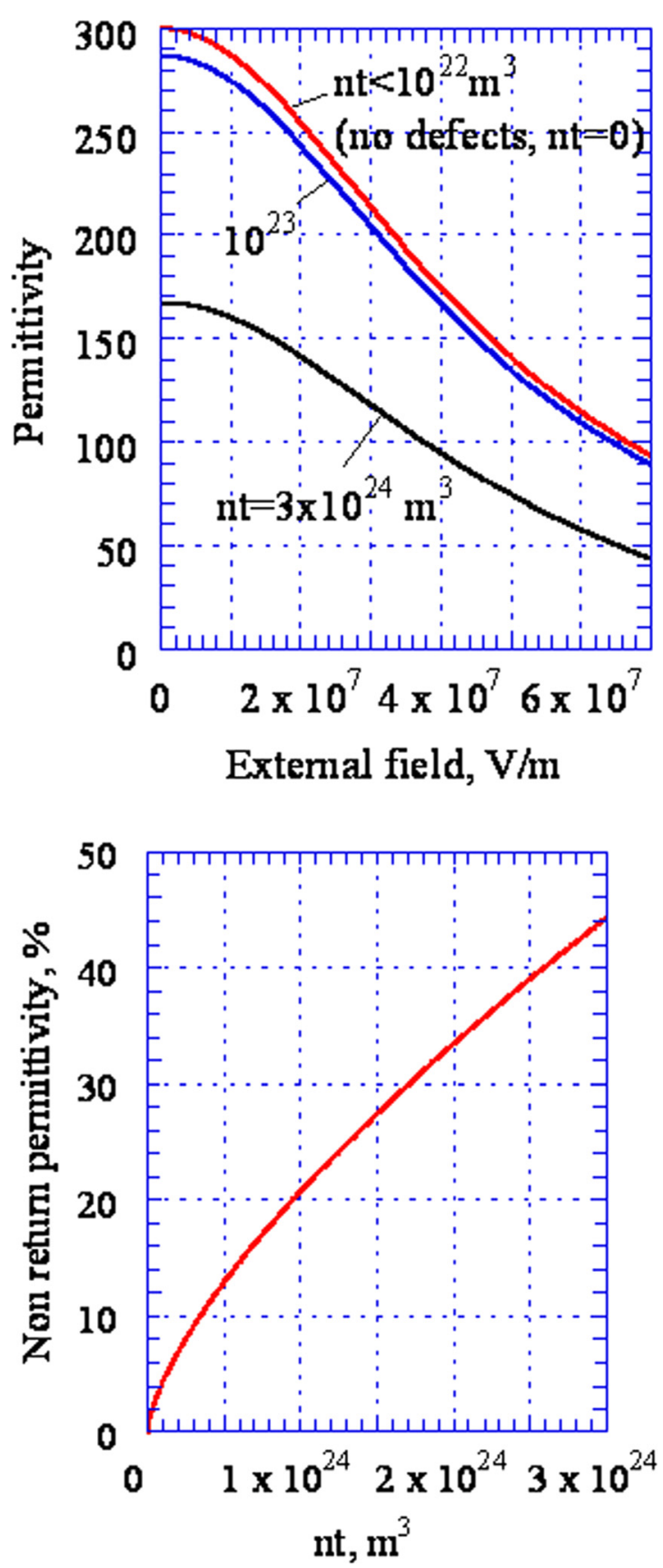

FIG. 10. (Color online) Apparent permittivity (a) and non-return permittivity $(\Delta \varepsilon \%)$ vs density of the charged defect.

using Eqs. (22) and (23). The density of the free electrons are assumed to be $n_{c}=10^{12} \mathrm{~m}^{-3}$ and $\mathrm{N}_{\mathrm{t}}=10^{24} \mathrm{~m}^{-3}$. The estimated density of the injected via Pt/STO barrier electrons is in the range of $10^{5}-10^{7} \mathrm{~m}^{-3}$ which is not sufficient for effective neutralization (trapping) of the charged defects. As it is seen, for the used density of the free electrons, almost all shallow traps are ionized already at very low fields while the deeper traps are only partially ionized even at higher voltages.

Shown in Fig. 12(a) is the dependence of the apparent permittivity on the depth of the trap, $\mathrm{W}_{\mathrm{t}}$, for a range of external fields and attempts to escape frequency $\nu=0.1$. All traps with $\mathrm{W}_{\mathrm{t}}<0.1 \mathrm{eV}$ release electrons at all bias voltages considered. In other words they are charged and reduce the

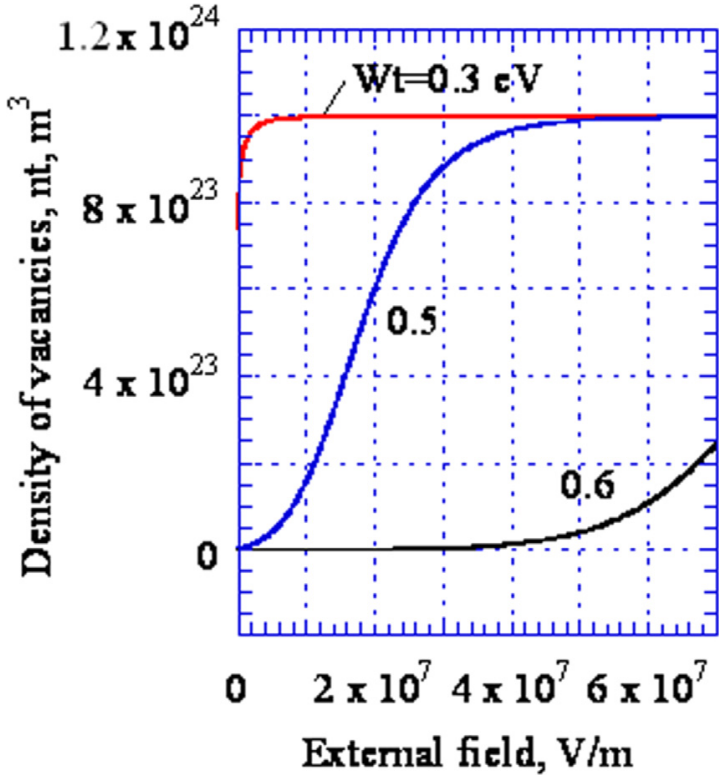

FIG. 11. (Color online) DC bias dependence of density of the charged traps for different trap energies.

apparent permittivity. With increased trap depth, higher voltages are needed for de-trapping. The electrons from the deeper traps, $\mathrm{W}_{\mathrm{t}}>0.6 \mathrm{eV}$, are not de-trapped and the permittivity is not affected, $\varepsilon_{\text {ap }}\left(\mathrm{E}_{\text {ext }}\right)=\varepsilon\left(\mathrm{E}_{\text {ext }}\right)$, i.e., it is governed by Eq. (3). The voltage dependencies of the apparent permittivity for different trap depths are shown in Fig. 11(b).

For intermediate trap energies (i.e., $\mathrm{W}_{\mathrm{t}}=0.4 \mathrm{eV}$, Fig. 12(b)) with increasing bias a gradual de- trapping takes place leading to a gradual reduction of the apparent permittivity. In this regard it is interesting to observe the increasing steepness of the $\varepsilon_{\mathrm{ap}}(\mathrm{E})$ in comparison to $\varepsilon(\mathrm{E})$, i.e., with an ideal crystal, i.e., for a sample with trap energy $0.4 \mathrm{eV}$, the PooleFrenkel effect driven de-trapping starts at about $15 \mathrm{~V} / \mu \mathrm{m}$ and completes at about $60 \mathrm{~V} / \mu \mathrm{m}$. Figures 13(a) and 13(b) depict correspondingly the trap energy and field dependencies of the apparent permittivity for $\nu=10$. In both $\nu=1$ and 10 cases the deeper $\left(\mathrm{W}_{\mathrm{t}}>0.6 \mathrm{eV}\right)$ traps remain neutral and do not contribute in the permittivity reduction at DC bias as high as $70 \mathrm{~V} / \mu \mathrm{m}$ while the steepness of the $\varepsilon_{\mathrm{ap}}\left(\mathrm{E}_{\mathrm{ext}}\right)$ dependence increases with increasing escape frequency $\nu$.

\section{B. Experimental evidence}

Figure 14 shows the cross section and the $\mathrm{C}-\mathrm{V}$ performance of a capacitor based on STO films grown on $\mathrm{MgO}$ substrates by laser ablation. The thickness of STO films is about $0.5 \mu \mathrm{m}$. Thin $\mathrm{Au}(0.5 \mu \mathrm{m}) / \mathrm{Ti}(0.015 \mu \mathrm{m})$ layers on top of the STO are used to form symmetric coplanar-plate capacitor structures. The gap width in the capacitors is $2.0 \mu \mathrm{m} .{ }^{19}$ Upon DC bias reversal the capacitance (i.e., the apparent permittivity) gradually decreases. After several cycles of the bias reversal the reduction "saturates" indicating that all electrons from the traps/defects are released. In this case the nonreturn capacitance, $\Delta \mathrm{C}$, is about $1 \%$.

Figure 15 shows the cross section and the $\mathrm{C}-\mathrm{V}$ performance of a parallel-plate $\mathrm{Pt} / \mathrm{STO} / \mathrm{Pt}$ capacitor. Platinized $(100 \mathrm{~nm}$ thick Pt) silicon is used as the substrate. STO films 

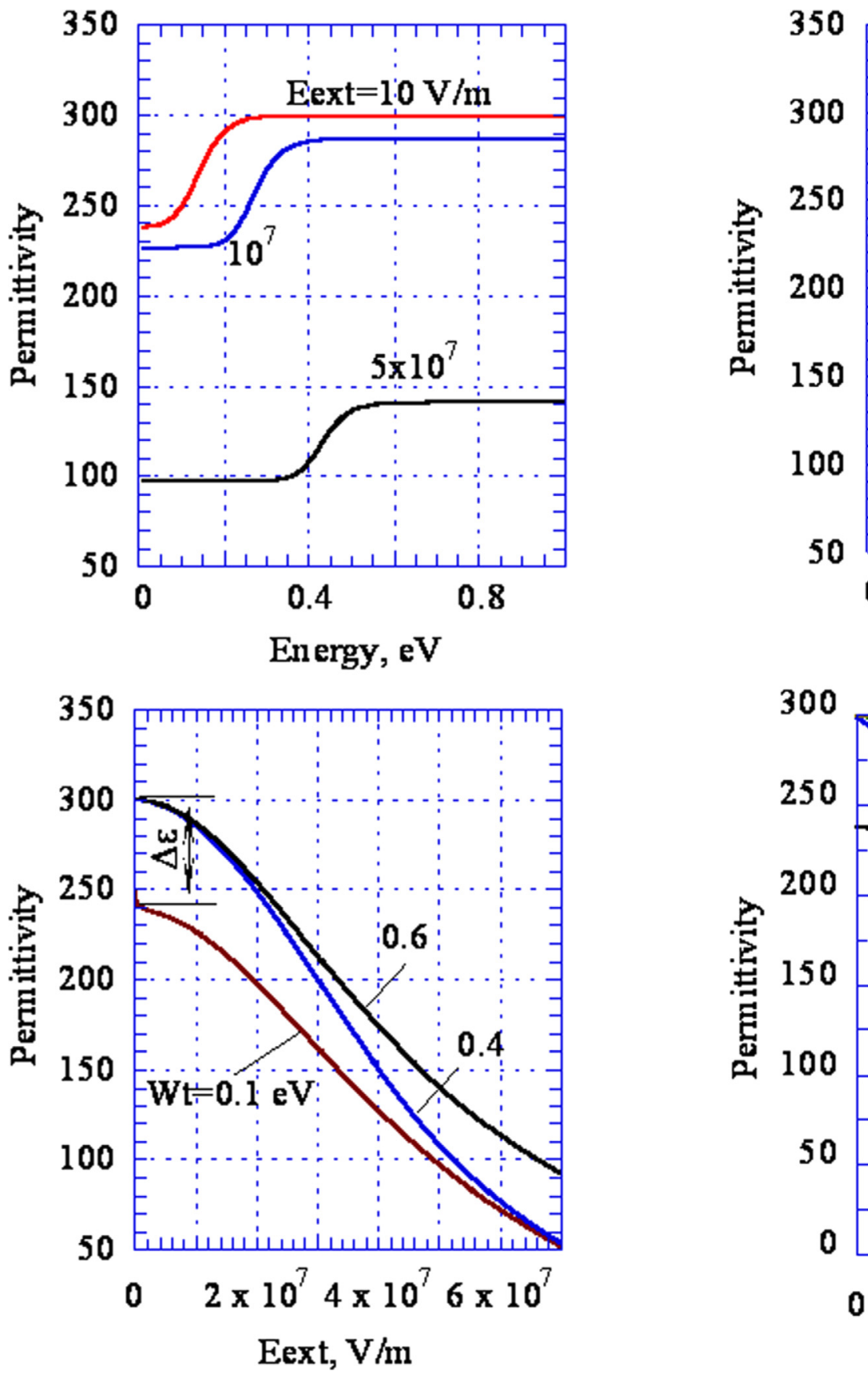

FIG. 12. (Color online) Apparent permittivity vs trap energy (a) and DC field (b). $\mathrm{N}_{\mathrm{t}}=10^{24} \mathrm{~m}^{-3}, \nu=0.1$.

( $300 \mathrm{~nm}$ thick) are grown by laser ablation of a $\mathrm{SrTiO}_{3}$ target. To keep the parallel-plate structure symmetric a $50 \mathrm{~nm}$ Pt layer is deposited on BST film by e-beam evaporation. However, due to the high temperature of deposition of STO film in oxygen ambient the bottom Pt/STO barrier is smeared out and is lower in comparison with the top Pt/STO interface. For this reason the electron injection from the bottom Schottky barrier is more intensive as compared with the top barrier leading to intensive trapping according to Eq. (18). As a result the number of the charged defects for negative voltages is less and thereby non-return capacitance associated with negative voltage branch is positive (close to zero). In contrast to the previous case no hysteresis is observed for

FIG. 13. (Color online) Apparent permittivity vs trap energy (a) and DC field (b). $\mathrm{N}_{\mathrm{t}}=10^{24} \mathrm{~m}^{-3}, \nu=10$. Shown are also permittivities $\varepsilon(\mathrm{E})$ of STO without defects, $\mathrm{n}_{\mathrm{t}}=0$, defined by Eq. (3).

high bias voltages (exceeding $15 \mathrm{~V}$ ). This indicates that for $\mathrm{V}>15 \mathrm{~V}$ all traps are charged and the apparent permittivity does not depend on the charged trap density (as for curves with $\mathrm{W}_{\mathrm{t}}<0.4 \mathrm{eV}$ and $\mathrm{V}>15 \mathrm{~V}$, Fig. 11(d)).

Figure 16 depicts measured $\mathrm{C}-\mathrm{V}$ and $\mathrm{I}-\mathrm{V}$ dependences of a $\mathrm{Pt} / \mathrm{Ba}_{0.25} \mathrm{Sr}_{0.75} \mathrm{TiO}_{3} / \mathrm{Pt}$ structure. The thickness of the ferroelectric film is $600 \mathrm{~nm}$, and the diameter of the top electrode is $30 \mu \mathrm{m}$. In a defectless ideal crystal the dielectric permittivity practically instantaneously follows the changes in the applied electric field according to Eq. (3). In a crystal with traps, additional reduction of the permittivity takes place according to Eq. (17) due to charging of the vacancies. 


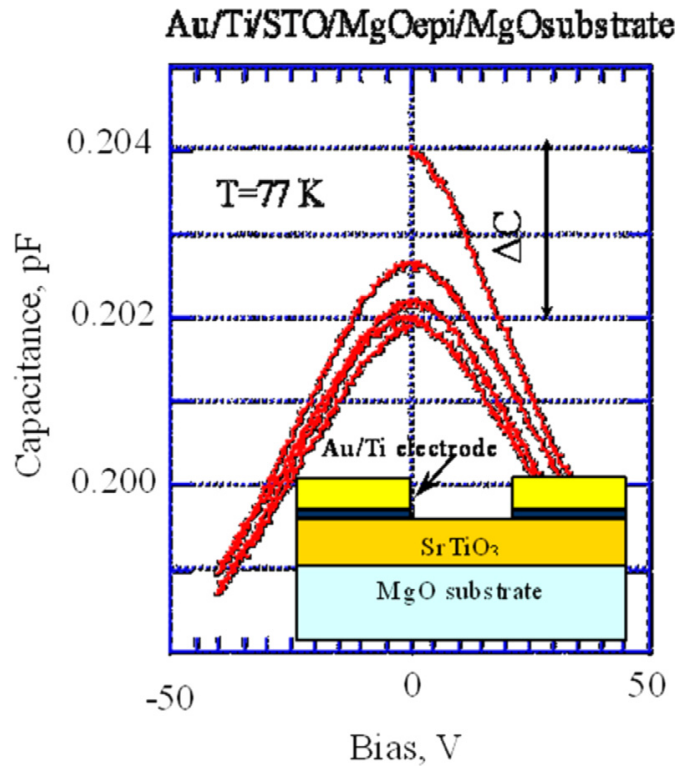

FIG. 14. (Color online) C-V dependence of a coplanar-plate STO capacitor.

The released due to Poole-Frenkel effect carriers, along with the injected via contacts carriers, contribute in the current in the external circuit. Trapping and de-trapping of the carriers is a dynamic process. With increasing field the number of the de-trapped carriers exceeds the number of trapped carriers resulting in an overall increase in the current. The reduction of the bias field, Fig. 16, is accompanied with increased trapping rate. However, the number of the charged (de-trapped) vacancies remains rather high since the trapping time is much longer than the scanning time of the bias field. As a result hysteresis appears in the $\mathrm{C}-\mathrm{V}$ and $\mathrm{I}-\mathrm{V}$ dependencies with a non-return capacitance $\Delta \mathrm{C}_{+}>0$. The current at $\mathrm{V}=0$ (no injection), Fig. 16, is due to de-trapped electrons. Similar hystereses are observed where the bias field is reversed. However, in this case the barrier height of the injecting bottom contact is lower (due to film growth process related, as

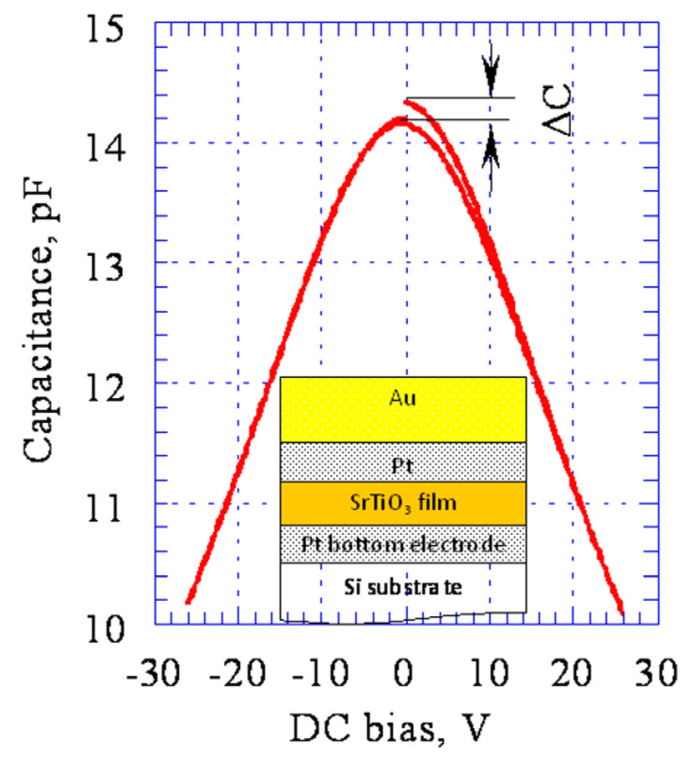

FIG. 15. (Color online) C-V performance of a parallel-plate STO based capacitor.

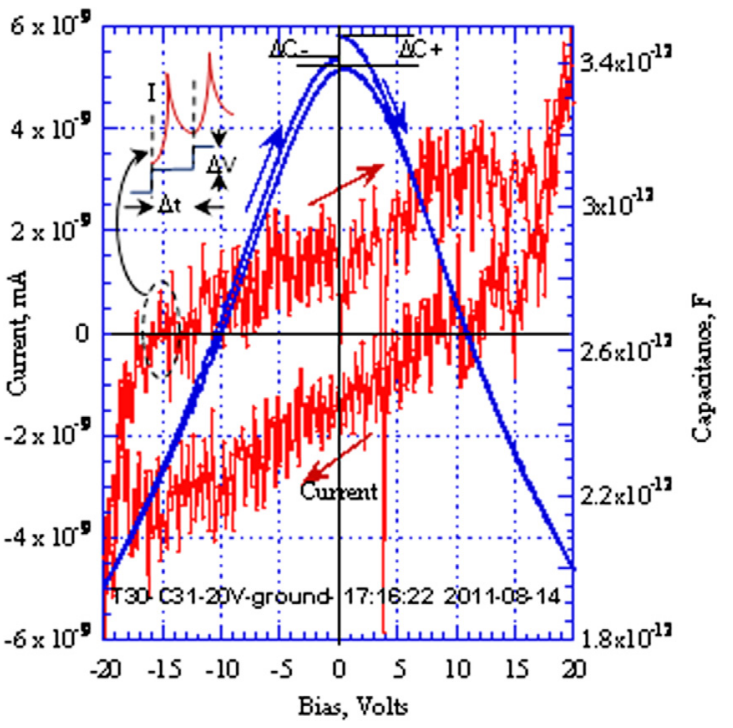

FIG. 16. (Color online) $\mathrm{C}-\mathrm{V}$ and $\mathrm{I}-\mathrm{V}$ dependencies of a $\mathrm{Pt} / \mathrm{Ba}_{0.25} \mathrm{Sr}_{0.75} \mathrm{TiO}_{3} /$ $\mathrm{Pt}$ structure. Arrows indicate the directions of changes in the capacitance and current.

described above), which results in more injected carriers and more intensive trapping. As a result the non-return capacitance, $\Delta \mathrm{C}_{-}<0$, and current at $\mathrm{V}=0$ are slightly higher. The pA scale oscillations in the current are associated with the stepwise changes in the bias voltage (see inset in Fig. 16) provided by the measurement instrument (HP4156B). Each $\Delta \mathrm{V}$ step releases some electrons increasing the current. In time interval $\Delta t$ some of the electrons are captured back and the current decreases.

\section{CONCLUSIONS}

The apparent permittivity approximation (17) is useful for the polarized cylindrical shape regions regardless their origin. In reality these regions may be associated with intrinsic defects and dopants. The intrinsic defects, such as vacancies of oxygen and strontium give traps with energies $0.4-05 \mathrm{eV} .{ }^{20}$ Some of the vacancies have relatively shallow traps $^{21}$ that are partially ionized even without external fields which (perhaps in combination with the interfacial dead layers) reduce the zero bias apparent permittivity of the thin films. The dopants, depending on their valency, act as neutral species, as acceptors, or as donors. ${ }^{22}$ In contrast to the bulk single crystal STO, the films are richer in defects, especially at the interfaces with the electrodes. ${ }^{1}$ The activation energies and the density of the traps depend not only their nature but also on the film fabrication processes, annealing condition, etc. The detailed study of the nature, density, and activation energies for a given ferroelectric film, ${ }^{23}$ taking interactions between the traps with different energies ${ }^{24}$ and, in general, the dynamics of trapping/de-trapping process is a subject for extended and dedicated studies and is beyond the scope of this paper. The reduction of the apparent permittivity considered in this work is only due to the charged traps in the bulk of paraelectric crystal. Any possible reduction associated with the interfacial "dead" layers and strain is not considered in this paper. In metal-paraelectric-metal (MPM) structures, 
such as varactors, the charged defects may be located in the bulk of the films and at the electrode-paraelectric interfaces. ${ }^{2}$ Distribution of the oxygen vacancies is not a constant across the film. Typically the density of the oxygen vacancies is higher in the layers interfacing with the electrodes. The dielectric theory of the charged defect proposed in this work may be extended for modeling the effects of the ferroelectric capacitors with interfacial layers. In a simple case an MPM structure may be represented by two interfacial layers with trap densities $\mathrm{N}_{\mathrm{t} 1}$ and $\mathrm{N}_{\mathrm{t} 2}$ sandwiching a middle layer with trap density $\mathrm{N}_{t}$. The effective dielectric properties of these layers may be given using the apparent permittivity model developed above.

\section{ACKNOWLEDGMENTS}

This work is supported by Grant No. 261 of the Ministry of Education and Science of Armenia and by the Swedish Research Council (Vetenskaprådet).

${ }^{1}$ W. A. Feil and B. W. Wessels, J. Appl. Phys. 74, 3927 (1993).

${ }^{2}$ M. Dawber, J. F. Rabe, and J. F. Scott, Rev. Mod. Phys. 77, 1083 (2005).

${ }^{3}$ O. G. Vendik, S. P. Zubko, and M. A. Nikol'ski, J. Appl. Phys. 92, 7448 (2002).

${ }^{4}$ S. Gevorgian, A. Vorobiev, and A. Deleniv, Ferroelectrics in Microwave Devices Circuits and Systems (Springer, London, 2009).
${ }^{5}$ A. I. Dedyk, S. F. Karmanenko, M. N. Malyshev, and L. T. Ter-Martirosyan, Phys. Solid State 37, 1906 (1995).

${ }^{6}$ A. Vorobiev, P. Rundqvist, K. Khamchane, and S. Gevorgian, J. Appl. Phys. 96, 4642 (2004).

${ }^{7}$ J. D. Baniecki, T. Shioga, K. Kurihara, and N. Kamehara, J Appl. Phys. 97, 114101 (2005).

${ }^{8}$ A. K. Tagantsev, I. Stolichnov, E. L. Colla, and N. Setter, J. Appl. Phys. 90, 1387 (2001).

${ }^{9}$ A. B. Kozyrev, V. N. Osadchy, D. M. Kosmin, and A. V. Tumarkin, Appl. Phys. Lett. 91, 022905 (2007).

${ }^{10}$ N. McN. Alford, P. Kr. Petrov, A. G. Gagarin, A. B. Kozyrev, A. I. Sokolov, O. I. Soldatenkov, and V. A. Volpyas, Appl. Phys. Lett. 87, 222904 (2005).

${ }^{11}$ T. Bieger, and J. Maler, Solid State Ionics 53-56, 578 (1992).

${ }^{12}$ R. I. Eglitis, E. A. Kotomin, and G. Borstel, Phys. Status Solidi C 2, 113 (2005).

${ }^{13}$ J. Gerblinger and H. Meixner, J. Appl. Phys. 67, 7453 (1990).

${ }^{14}$ D. C. Lupascu, Solid State Ionics 177, 3161 (2006).

${ }^{15}$ A. G. Milnes, Deep Impurities in Semiconductors (John Wiley \& Sons, New York, 1973), p. 558.

${ }^{16}$ A. K. Tagantsev and G. Gerra, J. Appl. Phys. 100, 051607 (2006).

${ }^{17}$ M. Stengel and N. A. Spaldin, Nature 443, 679 (2006).

${ }^{18}$ A. Antons, J. B. Neaton, K. M. Rabe, and D. Vanderbilt, Phys. Rev. B 71, 024102 (2005).

${ }^{19}$ S. Gevorgian, P. K. Petrov, S. Abadei, and Z. Ivanov, Integr. Ferroelectr. 33, 311 (2001).

${ }^{20}$ Y. -P. Wang and T. -Y. Tseng, J. Appl. Phys. 81, 6762 (1977).

${ }^{21}$ T. Tanaka, K. Matsunaga, Y. Ikuhara, and T. Yamamoto, Phys. Rev. B 68, 205213 (2003).

${ }^{22}$ J. Robertson, J. Appl. Phys. 93, 1054 (2003).

${ }^{23}$ M. O. Selme and P. Pecheur, J. Phys. C 21, 1779 (1988)

${ }^{24}$ C. Y. Chang, W. C. Hsu, S. J. Wang, and S. S. Hau, J. Appl. Phys. 60, 1042 (1986). 